\title{
ADAPTIVE CONTROL OF NONLINEAR VISUAL SERVOING SYSTEMS FOR 3D CARTESIAN TRACKING
}

\author{
Alessandro R. L. Zachi* \\ zachi@cefet-rj.br \\ Fernando Lizarralde ${ }^{\dagger}$ \\ fernando@coep.ufrj.br
}

\author{
Liu Hsu ${ }^{\dagger}$ \\ liu@coep.ufrj.br \\ Antonio C. Leite ${ }^{\dagger}$ \\ toni@coep.ufrj.br
}

\author{
${ }^{*}$ Department of Electrical Engineering \\ Centro Federal de Educação Tecnológica Celso Suckow da Fonseca - CEFET/RJ \\ Rio de Janeiro, RJ, Brazil \\ ${ }^{\dagger}$ Department of Electrical Engineering/COPPE \\ Federal University of Rio de Janeiro \\ Rio de Janeiro, RJ, Brazil
}

\begin{abstract}
This paper presents a control strategy for robot manipulators to perform 3D cartesian tracking using visual servoing. Considering a fixed camera, the 3D cartesian motion is decomposed in a $2 \mathrm{D}$ motion on a plane orthogonal to the optical axis and a 1D motion parallel to this axis. An image-based visual servoing approach is used to deal with the nonlinear control problem generated by the depth variation without requiring direct depth estimation. Due to the lack of camera calibration, an adaptive control method is used to ensure both depth and planar tracking in the image frame. The depth feedback loop is closed by measuring the image area of a target object attached to the robot end-effector. Simulation and experimental results obtained with a real robot manipulator illustrate the viability of the proposed scheme.
\end{abstract}

KEYWORDS: Adaptive Visual Servoing, Tracking, Depth Control, Uncertain Robotic Systems.

\footnotetext{
Artigo submetido em 28/02/2005

1a. Revisão em 10/04/2005

2a. Revisão em 16/08/2006

Aceito sob recomendação do Editor Associado

Prof. José Roberto Castilho Piqueira
}

\section{RESUMO}

Este trabalho apresenta uma estratégia de controle para robôs manipuladores realizarem rastreamento cartesiano 3D utilizando servovisão. Considerando uma câmera fixa, o movimento cartesiano 3D é decomposto em um movimento $2 \mathrm{D}$ sobre um plano ortogonal ao eixo óptico e em outro movimento $1 \mathrm{D}$ paralelo ao mesmo eixo. Uma abordagem de servovisão baseada em imagem é utilizada para tratar o problema de controle não-linear, gerado pela variação de profundidade, sem a necessidade de estimar esta medida. Devido à ausência de calibração da câmera, um método de controle adaptativo é utilizado para assegurar rastreamento planar e de profundidade nas coordenadas da imagem. A malha de controle de profundidade é fechada através da medição da área da imagem de um objeto fixado no efetuador do robô. Simulação e resultados experimentais, obtidos com um robô manipulador real, ilustram a viabilidade do esquema proposto.

PALAVRAS-CHAVE: Servovisão Adaptativa, Rastreamento, Controle de Profundidade, Sistemas Robóticos Incertos. 


\section{INTRODUCTION}

For many years researchers have been actively investigating the use of visual servoing in the control of robotic systems. The feedback provided by vision has been used to develop several control strategies with proven stability (c.f. Hutchinson et al. (1996)).

Solutions to the problem of robot motion control in a 3D environment were proposed with different choices of camera configurations, e.g., fixed (eye-to-hand) or moving camera (eye-in-hand) (Espiau et al., 1992; Corke and Hutchinson, 2000; Kelly et al., 2000; Conticelli and Allotta, 2001a). A restriction of some of these solutions is that they require direct estimate of the depth information with respect to the image frame. In Malis et al. (1999) an off-line learning stage is required to estimate the distance of the camera to the object of interest. In Fang et al. (2002), the off-line phase is not required to determine the unknown depth distance. Instead, an estimate is obtained at each interaction by means of an Euclidean homography method.

Other authors have considered the lack of depth information. For instance, Conticelli and Allotta (2001b) designed an adaptive kinematic controller to ensure uniformly ultimately bounded set-point regulation under some restrictions on the translational velocities and bounds on the uncertain depth parameter. Hsu et al. (2001) designed an adaptive controller to allow trajectory tracking on smooth surfaces non-orthogonal to the optical axis. Since the system model for visual servoing is nonlinear due to the depth displacement, an approximate linearly parameterized function representing the system was used in order to design a suitable linearly parameterized adaptive control law. The dependence of depth with respect to the $2 \mathrm{D}$ image coordinates was then adaptively compensated without measuring the depth.

In this work, a novel adaptive visual controller for cartesian robots using a fixed camera is developed in order to perform 3D tracking, when the camera calibration parameters are assumed uncertain. The main interest for compensating the lack of exact knowledge about the system parameters or environment, is to increase robot autonomy through sensorbased control without explicit human intervention or reprogramming. The cartesian motion is decomposed in a 2D motion on a plane orthogonal to the optical axis and a 1D motion parallel to this axis, which corresponds to the depth variation with respect to the image frame. An image-based visual servoing approach is used to deal with the nonlinear control problem generated by the varying depth without requiring its measurement.

The paper is organized as follows: Section 2 describes the tasks to be achieved and presents the basic description of the visual servoing model. In Section 3, an Image-Based approach is introduced and a depth MRAC controller is developed. The SDU method (Costa et al., 2003) is used in Section 4 to solve a 2D adaptive visual tracking control problem which accounts for the depth variation. Then, the overall system stability is analysed. Simulation results obtained with the proposed strategy are discussed in Section 5. In Section 6 the experimental results obtained with a robot manipulator are presented. Finally, conclusions are presented in Section 7.

\section{PROBLEM FORMULATION}

Consider the problem of controlling a robot manipulator to perform tracking on 3D environment, based on a desired image trajectory. As it can be seen in Figure 1, the key idea is to use vision feedback obtained from a fixed and uncalibrated camera to allow tracking along the $x, y, z$ directions with no measurement of the depth displacement.

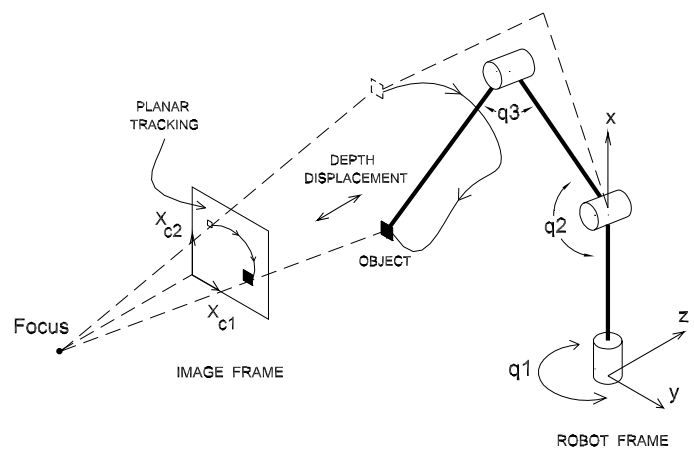

Figure 1: Depth and planar tracking.

Since motions are performed in a 3D environment, 3 degrees of freedom have to be controlled by the visual servoing system. Thus, at least three independent features need to be extracted from the image of a target object attached to the robot end-effector in order to accomplish a specified task. In this paper, the image centroid will be used to provide stable tracking control of the robot with respect to a desired image trajectory. Simultaneously, the image area will be extracted to provide depth tracking. Although those two tasks might interact significantly, we will show in the following that they are in fact only partially coupled. This facilitates the controller design.

\subsection{D planar subsystem}

Let $(X, Y, Z)$ be the coordinates of an object centroid projected in the camera frame and $(x, y, z)$ be the coordinates of the object centroid in the base frame of the robot. Here, we 
assume that the camera and robot frames have the same orientation with affine $z$-axis. The two coordinates are related as follows:

$$
\left[\begin{array}{l}
X \\
Y \\
Z
\end{array}\right]=R(\phi)\left[\begin{array}{l}
x \\
y \\
z
\end{array}\right]+T
$$

where $R(\phi) \in S O(3)$ is an elementary rotation matrix around the $z$-axis, which considers the camera misalignment angle $\phi$ with respect to the base frame, and $T$ is a translational vector. Using a pinhole model of the camera with focal length $f$ and meter-to-pixel scale factors $\alpha_{i}(i=1,2)$, the coordinates of the object centroid in the image frame are given by

$$
\left[\begin{array}{l}
x_{c 1} \\
x_{c 2}
\end{array}\right]=\frac{f}{Z}\left[\begin{array}{cc}
\alpha_{1} & 0 \\
0 & \alpha_{2}
\end{array}\right]\left[\begin{array}{l}
X \\
Y
\end{array}\right]
$$

The differential kinematics relationship in the image frame is established by

$$
\left[\begin{array}{c}
\dot{x}_{c 1} \\
\dot{x}_{c 2}
\end{array}\right]=\frac{1}{Z}\left[\begin{array}{ccc}
f \alpha_{1} & 0 & -x_{c 1} \\
0 & f \alpha_{2} & -x_{c 2}
\end{array}\right]\left[\begin{array}{c}
\dot{X} \\
\dot{Y} \\
\dot{Z}
\end{array}\right]
$$

that is,

$$
\left[\begin{array}{c}
\dot{x}_{c 1} \\
\dot{x}_{c 2}
\end{array}\right]=\frac{1}{Z}\left[\begin{array}{ccc}
f \alpha_{1} & 0 & -x_{c 1} \\
0 & f \alpha_{2} & -x_{c 2}
\end{array}\right] R(\phi)\left[\begin{array}{c}
\dot{x} \\
\dot{y} \\
\dot{z}
\end{array}\right]
$$

In this paper, we assume that the robot is purely kinematic and that the end-effector velocity can be directly controlled, that is, $v=\left[\begin{array}{lll}\dot{x} & \dot{y} & \dot{z}\end{array}\right]^{T}$, where $v$ is the control variable to be designed.

\subsection{D depth subsystem}

For the depth description, we need to make the following assumptions:

Assumption 1 The motions in the 3D environment are such that the object attached to the robot end-effector is planar and always parallel to the image frame.

Assumption 2 The object projected surface $S_{c}$ is assumed to be within the image range $0<S_{\min }<S_{c}<S_{\max }$ for all $t$.

The dynamics of the object projected surface $S_{c}$, expressed in the image frame, is described by (Flandin et al., 2000; Zachi et al., 2004)

$$
\dot{S}_{c}=-\left(\frac{2 S_{c}}{Z}\right) \dot{Z}
$$

\subsection{Complete translational model}

Since from (1) $\dot{Z}=\dot{z}$, the overall kinematic model of the planar/depth system is given by

$$
\left[\begin{array}{c}
\dot{x}_{c 1} \\
\dot{x}_{c 2} \\
\dot{S}_{c}
\end{array}\right]=\frac{1}{Z} \underbrace{\left[\begin{array}{ccc}
f \alpha_{1} & 0 & -x_{c 1} \\
0 & f \alpha_{2} & -x_{c 2} \\
0 & 0 & -2 S_{c}
\end{array}\right]}_{L_{0}\left(s_{T}\right)} R(\phi)\left[\begin{array}{c}
\dot{x} \\
\dot{y} \\
\dot{z}
\end{array}\right] .
$$

Now, let $s_{T}=\left[\begin{array}{ll}x_{c}^{T} & S_{c}\end{array}\right]^{T}$ be the image feature vector and $v=\left[\begin{array}{lll}\dot{x} & \dot{y} & \dot{z}\end{array}\right]^{T}$ be the translational velocity vector in the robot frame, the model (6) can be rewritten as

$$
\dot{s}_{T}=\frac{1}{Z} L_{T}\left(s_{T}\right) v,
$$

with

$$
\begin{aligned}
L_{T}\left(s_{T}\right) & =L_{0}\left(s_{T}\right) R(\phi) \\
& =\left[\begin{array}{ccc}
f \alpha_{1} \cos (\phi) & -f \alpha_{1} \sin (\phi) & -x_{c 1} \\
f \alpha_{2} \sin (\phi) & f \alpha_{2} \cos (\phi) & -x_{c 2} \\
0 & 0 & -2 S_{c}
\end{array}\right],
\end{aligned}
$$

where the matrix $L_{T}\left(s_{T}\right)$ is also known as image Jacobian (Hutchinson et al., 1996). In what follows, we will show that by extracting the object projected surface from the image, a cartesian controller can be designed even when a direct measure of $Z$ is not available.

\section{ADAPTIVE DEPTH TRACKING}

In this section, the goal is to design an adaptive control law that drives the system (7) to a specific depth position in accordance to a known desired image projected surface $S_{c}^{*}$. Note from (7) that $\dot{z}$ is the only control variable which interacts with $S_{c}$ and thus, a scalar control strategy can be adopted for the depth tracking problem.

Let $S_{c_{0}}$ denote a known surface corresponding to some depth $Z_{0}$. Then, we can integrate both sides of (5) to obtain the following relationship (Zachi et al., 2004):

$$
Z=Z_{0}\left(\frac{S_{c_{0}}}{S_{c}}\right)^{\frac{1}{2}}
$$

From (7) and (8), we define a scaled version of the translational velocity vector $v$

$$
W=\left[\begin{array}{l}
w_{1} \\
w_{2} \\
w_{3}
\end{array}\right]=\left(\frac{S_{c}}{S_{c_{0}}}\right)^{\frac{1}{2}} v
$$

where $S_{c}$ is continuously captured by the camera. Then, rewriting $\dot{S}_{c}$ based on (7), (8) and (9), we finally obtain the following affine system model:

$$
\dot{S}_{c}=k_{p} S_{c} w_{3},
$$


with

$$
k_{p}=-2 / Z_{0} .
$$

Assumption $3 Z_{0}$ is assumed positive, but otherwise unknown.

\subsection{MRAC design}

Since the dynamics of $S_{c}$, in the third row of (7), is only affected by $\dot{z}$, an adaptive controller can be designed via standard Model Reference Adaptive Control (MRAC) method (Ioannou and Sun, 1996).

A simple reference model for this subsystem, in terms of a reference projected surface $S_{c}^{*}$, is given by

$$
\dot{S}_{c_{M}}=-\lambda_{m} S_{c_{M}}+\lambda_{m} S_{c}^{*}, \quad \lambda_{m}>0 .
$$

In this case, the ideal signal $w_{3}^{*}$ which provides perfect matching between (10) and (12), is given by

$$
\begin{gathered}
w_{3}^{*}=\theta_{3}^{*} \xi, \\
\theta_{3}^{*}=\frac{\lambda_{m}}{k_{p}}, \quad \xi=\frac{S_{c}^{*}-S_{c}}{S_{c}} .
\end{gathered}
$$

Using the certainty equivalence principle (Ioannou and Sun, 1996), we set

$$
w_{3}=\theta_{3} \xi,
$$

which leads to the following closed loop error equation:

$$
\dot{e}_{s}=-\lambda_{m} e_{s}+k_{p} \tilde{\theta}_{3} \xi
$$

where $e_{s}=S_{c}-S_{c_{M}}$ is the depth tracking error and $\tilde{\theta}_{3}=$ $\theta_{3}-\theta_{3}^{*}$ is the parametric error. From the standard adaptive control theory, if the sign of $k_{p}$ is known, then an adaptation law that guarantees asymptotic convergence of $e_{s}(t)$ and the uniform boundedness of the system signals, is given by

$$
\dot{\tilde{\theta}}_{3}=\gamma_{3} e_{s} \xi, \quad \gamma_{3}>0 .
$$

To prove it, the following Lyapunov function is used

$$
V_{s}\left(e_{s}, \tilde{\theta}_{3}\right)=\frac{1}{2}\left(e_{s}^{2}+\gamma_{3}^{-1}\left|k_{p}\right| \tilde{\theta}_{3}^{2}\right) .
$$

The time derivative of (17) along (15) is given by

$$
\dot{V}_{s}\left(e_{s}, \tilde{\theta}_{3}\right)=-\lambda_{m} e_{s}^{2}+e_{s} k_{p} \tilde{\theta}_{3} \xi+\gamma_{3}^{-1}\left|k_{p}\right| \tilde{\theta}_{3} \dot{\tilde{\theta}}_{3},
$$

which by virtue of (16), leads to

$$
\dot{V}_{s}\left(e_{s}, \tilde{\theta}_{3}\right)=-\lambda_{m} e_{s}^{2} \leq 0 .
$$

The boundedness properties of the closed loop system signals are demonstrated from (17) and (19). By differentiating (19), we can verify that $\ddot{V}_{s}\left(e_{s}, \tilde{\theta}_{3}\right)$ is bounded and finally conclude, from Barbalat's Lemma, that $\lim _{t \rightarrow \infty} e_{s}(t) \rightarrow 0$.

\section{ADAPTIVE PLANAR TRACKING}

Once we have shown that the end-effector can be properly positioned by the MRAC controller developed in the previous section, our goal now is to perform asymptotic tracking of some predefined image trajectory. However, as can be observed from (7), both $x_{c 1}$ and $x_{c 2}$ interact with all the components of the scaled control $W$ generating a coupled multivariable subsystem. Indeed, reproducing the first two rows of the nonlinear system (7), also based on (8) and (9), we have

$$
\dot{x}_{c}=K_{T} u+G_{T} w_{T},
$$

with

$$
\begin{gathered}
K_{T}=\frac{f}{Z_{0}}\left[\begin{array}{rr}
\alpha_{1} \cos (\phi) & -\alpha_{1} \sin (\phi) \\
\alpha_{2} \sin (\phi) & \alpha_{2} \cos (\phi)
\end{array}\right], \quad u=\left[\begin{array}{l}
w_{1} \\
w_{2}
\end{array}\right], \\
G_{T}=\frac{1}{Z_{0}}, \quad w_{T}=w_{3}\left[\begin{array}{l}
x_{c 1} \\
x_{c 2}
\end{array}\right],
\end{gathered}
$$

which is a linearly parameterized plant. Then, an adequate control parameterization must follows since now we are dealing with matrix control gains instead of a scalar gain. Some works have gone toward this issue (Ioannou and Sun, 1996), however assuming restrictive conditions and/or conditions very difficult to satisfy in practice (a detailed discussion about such conditions can be found in Hsu and Costa (1999)). Most recent methods have proven to be less restrictive (Hsu and Costa, 1999; Costa et al., 2003; Ortega et al., 2003). Here, we will adopt the one introduced in Costa et al. (2003), which uses a Symmetric-Diagonal-Upper (SDU) factorization of the system gain matrix.

\subsection{Control design}

In this section, the control design will follow the one in Zachi et al. (2004). For the subsystem (20), consider the following reference model:

$$
\begin{aligned}
\dot{x}_{c_{M}} & =-\lambda_{M} x_{c_{M}}+\lambda_{M} r_{c}(t), \\
y_{c_{M}} & =x_{c_{M}},
\end{aligned}
$$

where $\lambda_{M}>0$ and $r_{c}(t) \in \Re^{2}$ is a bounded exogenous reference signal. The ideal control signal $u=u^{*}$ that perfectly matches (20) and (21), is given by

$$
u^{*}=K_{T}^{-1}\left[\lambda_{M}\left(r_{c}-x_{c}\right)-G_{T} w_{T}\right],
$$

which can be written as $u^{*}=P^{*} \sigma$ with

$$
\begin{aligned}
& P^{*}=\left[\begin{array}{llll}
p_{11}^{*} & p_{12}^{*} & p_{13}^{*} & p_{14}^{*} \\
p_{21}^{*} & p_{22}^{*} & p_{23}^{*} & p_{24}^{*}
\end{array}\right], \\
& \sigma=\left[\begin{array}{ll}
\left(r_{c}-x_{c}\right)^{T} & w_{T}^{T}
\end{array}\right]^{T} .
\end{aligned}
$$

Since $P^{*}$ is an uncertain matrix, we again use the certainty equivalence principle to design $u$ as

$$
u=P \sigma,
$$


where $P$ is the matrix of the adaptive parameters $p_{i j}$. Thus, to obtain the closed loop error equation we first add and subtract (23) in (20), that is,

$$
\begin{aligned}
\dot{x}_{c} & =K_{T} u+G_{T} w_{T}+K_{T} u^{*}-K_{T} u^{*} \\
& =-\lambda_{M} x_{c}+\lambda_{M} r_{c}+K_{T}\left(u-u^{*}\right) .
\end{aligned}
$$

Denoting $\tilde{u}=u-u^{*}$ and the planar tracking error by $e_{c}=$ $x_{c}-x_{c_{M}}$, we subtract (21) from (27) and finally obtain

$$
\dot{e}_{c}=-\lambda_{M} e_{c}+K_{T} \tilde{u} .
$$

\subsection{Parameterization via SDU factoriza- tion}

According to Costa et al. (2003), if $K_{T}$ has non-zero leading principal minors, then it is always possible to factorize $K_{T}$ as

$$
K_{T}=S D U
$$

where $S$ denotes a symmetric and positive definite matrix, $D$ denotes a diagonal matrix and $U$ an unitary upper triangular one.

Assumption 4 The sign of the leading principal minors of $K_{T}$ are known.

Then, from (27) and (29) we can write

$$
\begin{aligned}
\dot{e}_{c} & =-\lambda_{M} e_{c}+S D U\left(u-P^{*} \sigma\right) \\
& =-\lambda_{M} e_{c}+S D\left(U u-U P^{*} \sigma\right)
\end{aligned}
$$

Here, employing the decomposition $U u=u-(I-U) u$ we also have

$$
\dot{e}_{c}=-\lambda_{M} e_{c}+S D[u-\Lambda \sigma-(I-U) u],
$$

with $\Lambda=U P^{*}$. If we introduce a new ideal control vector

$$
\left[\begin{array}{c}
\Theta_{1}^{* T} \Omega_{1} \\
\Theta_{2}^{* T} \Omega_{2}
\end{array}\right] \equiv \Lambda \sigma+(I-U) u
$$

where $\Omega_{1}=\left[\begin{array}{ll}\sigma^{T} & u_{2}\end{array}\right]^{T}$ and $\Omega_{2}=\sigma$, the closed loop error equation (31) reduces to

$$
\dot{e}_{c}=-\lambda_{M} e_{c}+S D\left(u-\left[\begin{array}{c}
\Theta_{1}^{* T} \Omega_{1} \\
\Theta_{2}^{* T} \Omega_{2}
\end{array}\right]\right),
$$

from which we can extract the final control parameterization

$$
u=\left[\Theta_{1}^{T} \Omega_{1} \quad \Theta_{2}^{T} \Omega_{2}\right]^{T} .
$$

\subsection{Adaptation laws}

Based on the Assumption 4 and the factorization properties discussed in (Costa et al., 2003), we conclude that the entries of $D=\operatorname{diag}\left\{d_{1}, d_{2}\right\}$ have known signs. Since $S$ is a symmetric and positive definite matrix, we follow the standard Lyapunov design by choosing the following Lyapunov function candidate

$$
2 V_{c}\left(e_{c}, \tilde{\Theta}\right)=e_{c}^{T} S^{-1} e_{c}+\gamma^{-1} \sum_{i=1}^{2}\left(\left|d_{i}\right| \tilde{\Theta}_{i}^{T} \tilde{\Theta}_{i}\right) .
$$

The time derivative of (35) along (33) yields

$$
\begin{aligned}
\dot{V}_{c}\left(e_{c}, \tilde{\Theta}\right)= & -\lambda_{M} e_{c}^{T} S^{-1} e_{c}+\sum_{j=1}^{2}\left(e_{c j} d_{j} \tilde{\Theta}_{j}^{T} \Omega_{j}\right)+ \\
& \gamma^{-1} \sum_{i=1}^{2}\left(\left|d_{i}\right| \tilde{\Theta}_{i}^{T} \dot{\tilde{\Theta}}_{i}\right)
\end{aligned}
$$

Then, by choosing the adaptation laws as

$$
\dot{\tilde{\Theta}}_{i}=-\gamma \operatorname{sign}\left(d_{i}\right) e_{c_{i}} \Omega_{i},(i=1,2),
$$

equation (36) reduces to

$$
\dot{V}_{c}\left(e_{c}, \tilde{\Theta}\right)=-\lambda_{M} e_{c}^{T} S^{-1} e_{c} \leq 0 .
$$

From (35) and (38), we conclude that $e_{c}(t), \tilde{\Theta}(t) \in \mathcal{L}_{\infty}$, which implies that $x_{c}(t), \Theta(t) \in \mathcal{L}_{\infty}$. From the boundedness properties of (25), (33) and (34), we verify that the second derivative of $V_{c}\left(e_{c}, \tilde{\Theta}\right)$ is also bounded. Then, by using the Barbalat's Lemma, we can conclude that $\lim _{t \rightarrow \infty} e_{c}(t) \rightarrow 0$. Thus, the convergence and boundedness properties of all the closed loop signals can be demonstrated.

The following theorem states the stability properties of the overall visual servoing system.

Theorem 1 Consider the adaptive visual servoing system composed by (7), reference models (12) and (21), control laws (14) and (34), and adaptation laws (16) and (37). If Assumptions 1-4 are satisfied and the camera misalignment angle $\phi \in\left(-\frac{\pi}{2}, \frac{\pi}{2}\right)$ then: (a) all the closed loop signals are uniformly bounded; (b) for $e(t):=\left[\begin{array}{ll}e_{c}^{T} & e_{s}\end{array}\right]^{T}, e(t) \in \mathcal{L}_{2} \bigcap \mathcal{L}_{\infty}$, $\lim _{t \rightarrow \infty} e(t) \rightarrow 0$.

Proof: From (17) and (35), define $V=V_{s}+V_{c}$ which is positive definite. Thus $\dot{V} \leq 0$ follows that all signal are uniformly bounded and from the Barbalat's Lemma $e(t) \in$ $\mathcal{L}_{2} \bigcap \mathcal{L}_{\infty}, \lim _{t \rightarrow \infty} e(t) \rightarrow 0$.

Remark 1 Note that, the cartesian control $v$ can be recovered from (14) and (34) using the equation (9). 
Remark 2 (General Robotic Systems) In the previous sections, the key reason to adopt Assumption 1 was to exclude rotational motions of the object, ensuring that its projected surface only changes with depth. Indeed, it is well-known from Haralick and Shapiro (1993),page 50, that non-affine rotations of a planar object relatively to the camera optical axis modify its image projected surface.

It is important to emphasize that the Assumption 1 is violated, for general robotic systems, when object rotations are performed. Then, relation (8) would no longer hold. However, if a spherical object (Figure 2) is used instead of the planar one, it is possible to broaden the proposed strategy to consider the more general case. Indeed, adopting this particular object, the image projected surface becomes invariant with respect to the object rotation. Note that in this case relation (8) can still be used, at least for motions not too far away from the optical axis.

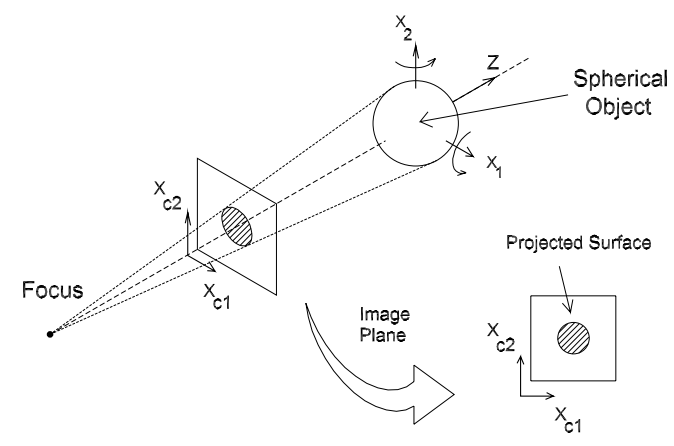

Figure 2: Image projection of a spherical object.

\section{SIMULATION RESULTS}

In order to illustrate the performance of the proposed adaptive scheme, we present simulation results with a 3DOF robot manipulator. The parameters of the visual servo system are: $f=6.0[\mathrm{~mm}], \phi=\pi / 6[\mathrm{rad}], \alpha_{1}=\alpha_{2}=83.0[\mathrm{pixel} / \mathrm{mm}]$. Also we consider $S_{c 0}=1$ [pixel] for $Z_{0}=1.0[\mathrm{~m}]$ and $S_{c}^{*}=1$ [pixel]. For the adaptive controllers (14),(16) and (34),(37), we have set $\gamma_{3}=0.4, \lambda_{m}=1.0$ and $\gamma=20.0$, $\lambda_{M}=2.0$ respectively. Other simulation parameters are set to: $\omega_{n}=0.5[\mathrm{rad} / \mathrm{s}] ; r_{c}(t)=\left[\sin \left(\omega_{n} t\right) \cos \left(\omega_{n} t\right)\right]^{T}$; $\theta_{3}(0)=0 ; \Theta_{1}(0)=\left[\begin{array}{lllll}0 & 0 & 0 & 0 & 0\end{array}\right]^{T} ; \Theta_{2}(0)=\left[\begin{array}{llll}0 & 0 & 0 & 0\end{array}\right]^{T}$.

The system behavior is presented in Figures 3-8. The asymptotic convergence of image tracking errors (planar and depth) can be observed in Figure 3.

The parameters behavior is illustrated in Figure 4, where it can be observed that the parameters tend to a steady state

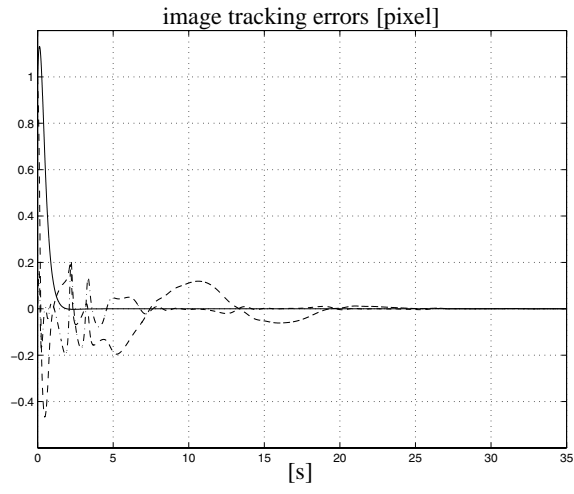

Figure 3: Image tracking errors: $e_{c 1}(-\cdot-) ; e_{c 2}(--) ; e_{s}(-)$.

value after a adaptation period. The cartesian control signals can be considered adequate and they are depicted in Figure 5. The depth variation due to the tracking control is illustrated in Figure 6. The tracking of trajectory in the image frame is illustrated in Figure 7. Note that the tracking is well behaved despite the presence of input disturbances introduced by the depth control. Figure 8 shows the end-effector behavior in the $3 \mathrm{D}$ environment.

\section{EXPERIMENTAL RESULTS}

In this section, we present experimental results obtained by the implementation of the adaptive visual tracking controller proposed in Sections 3 and 4.

\subsection{Workspace}

The experiments are performed on a 6DOF Zebra Zero manipulator (IMI Inc.). A KPD-50 CCD camera (Hitachi Ltd.), with a lens of focal length $f=6.0[\mathrm{~mm}]$, is mounted in front of the robot (see Figure 9 for a camera point of view). The extracted visual features are the image coordinates of a white sphere centroid located at the robot wrist and its image projected surface. The images of $640 \times 480$ [pixel] are acquired using a Meteor frame-grabber (Matrox Ltd.) at 30 frames per second (FPS) with 256 grey levels. The image processing is performed on a $50 \times 50$ [pixel] sub-window, in order to guarantee that the sphere remains within the subwindow. The first estimations of the white sphere coordinates and area are performed off-line using a Graphical User Interface (Figure 9), named VServo, developed in Tcl/Tk language. During task execution, features (centroid and area) are computed using the image moments algorithm (Haralick and Shapiro, 1993).

The visual servo controller is coded in C language and executed on a Pentium 200 SBC running Linux OS at 35.0 [ $\mathrm{ms}]$. 


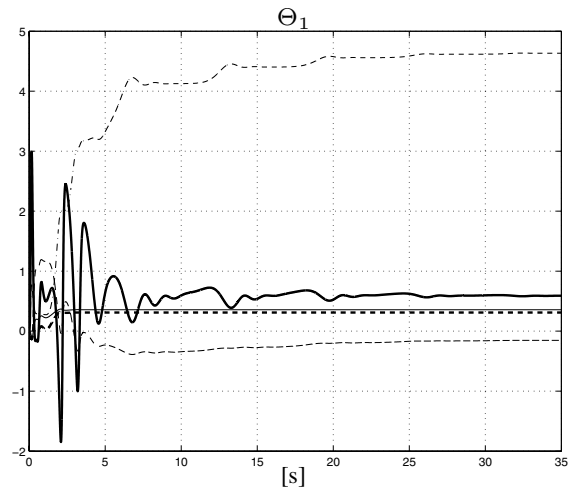

$\Theta_{2}$
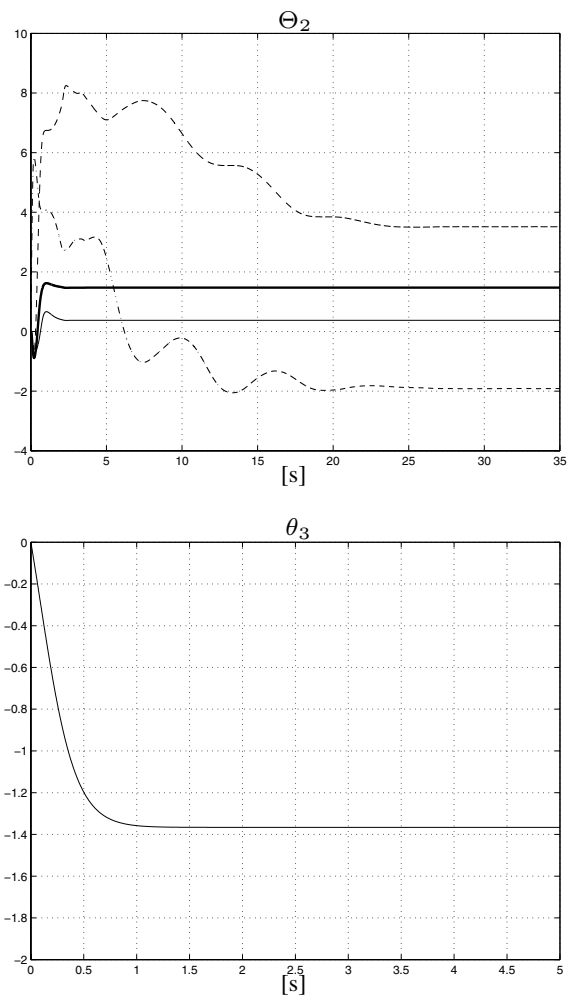

Figure 4: Adaptive parameters: $\Theta_{1}, \Theta_{2}$ and $\theta_{3}$.

The joint velocity command generated by the adaptive control law feeds the Zebra Zero (ISA board) which closes the velocity loop using an HCTL1100 microcontroller (HP Inc.) working in proportional velocity mode at $0.52[\mathrm{~ms}]$.

Due to noise sensitivity, the proportional gain in the velocity loop is not high enough to eliminate steady state error due to gravity effect. This disturbance is identified off-line (using a least square method) and effectively compensated.

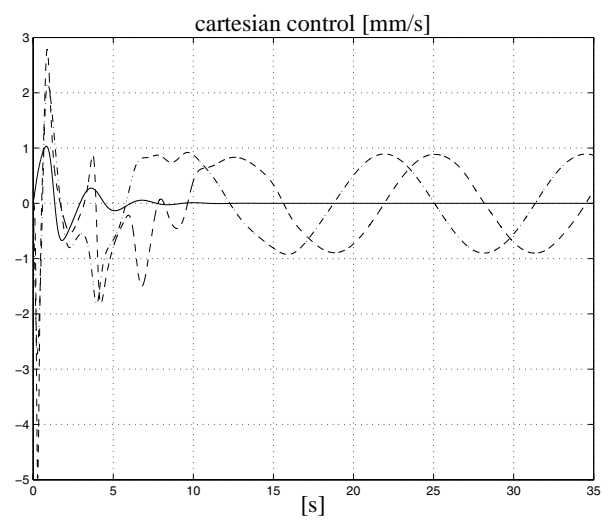

Figure 5: Control signals: $v_{1}(-\cdot) ; v_{2}(--) ; v_{3}(-)$.

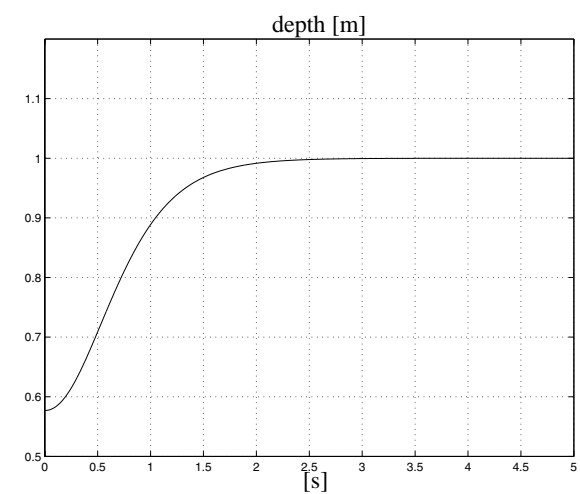

Figure 6: Depth behavior.

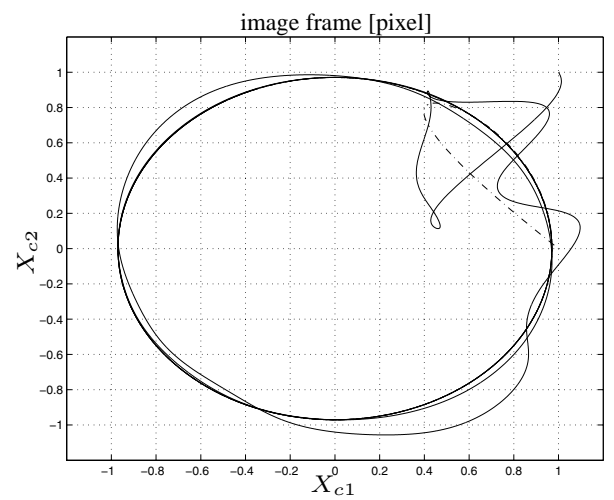

Figure 7: Trajectories: $x_{c_{M}}(-\cdot-) ; x_{c}(-)$.

\subsection{Analysis of Results}

The experimental tests are performed without regarding any calibration procedure. The camera parameters are: $\phi \approx$ $0, f=6.0[\mathrm{~mm}], \alpha_{1}=119[\mathrm{pixel} / \mathrm{mm}]$ and $\alpha_{2}=102$ 


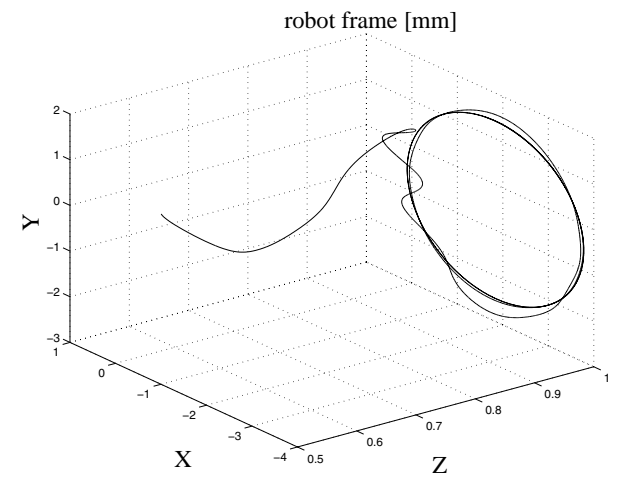

Figure 8: End-effector trajectory.

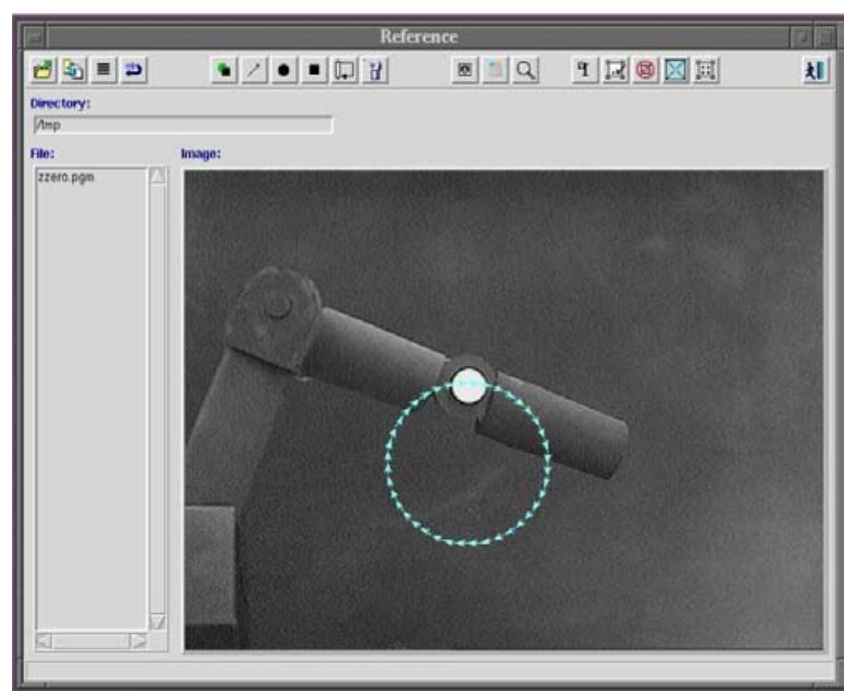

Figure 9: Experimental setup.

[pixel $/ \mathrm{mm}$ ]. The initial conditions for the adaptive parameters are $\theta_{3}(0)=0 ; \Theta_{1}(0)=\left[\begin{array}{lllll}0 & 0 & 0 & 0 & 0\end{array}\right]^{T} ; \Theta_{2}(0)=$ $\left[\begin{array}{llll}0 & 0 & 0 & 0\end{array}\right]^{T}$. The control parameters are $\gamma_{3}=5 \times 10^{-3}$, $\lambda_{m}=1.0, \gamma=2 \times 10^{-3}$ and $\lambda_{M}=1.0$ respectively. Other parameters are: $S_{c 0}=860$ [pixel] for $Z_{0}=1.0[\mathrm{~m}]$ and $S_{c}^{*}=700$ [pixel].

Figure 10 shows the tracking errors $e_{c}$ and $e_{s}$. It can be observed that $e_{c}$ and $e_{s}$ tends to small residual regions of orders 4 [pixel] and 10 [pixel] respectively. Figure 11 presents the time history of the centroid position and the projected surface in the image frame. The cartesian control signal and the joint control signal are depicted in Figure 12. The tracking of trajectory in the image frame and the end-effector trajectory described in the workspace are shown in Figures 13 and 14 respectively.
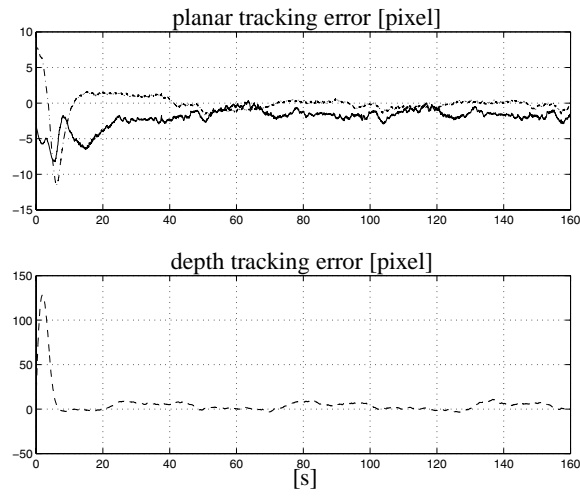

Figure 10: Image errors: $e_{c 1}(-\cdot) ; e_{c 2}(--) ; e_{s}(-)$.

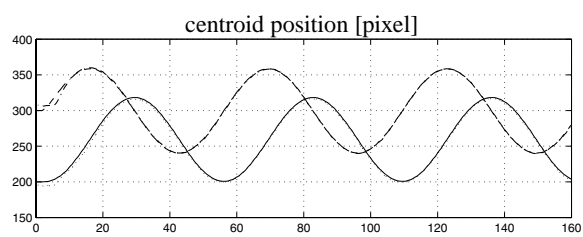

projected surface [pixel]

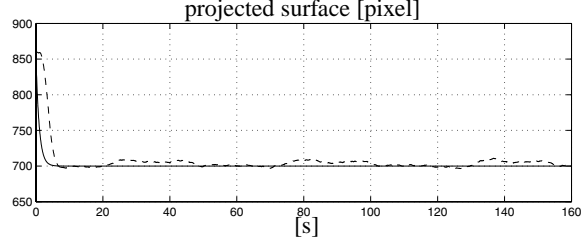

Figure 11: $x_{c 1}(-\cdot-), x_{c d 1}(--), ; x_{c 2}(\cdot), x_{c d 2}(-) ; S_{d}(-)$, $S_{c}(-)$.
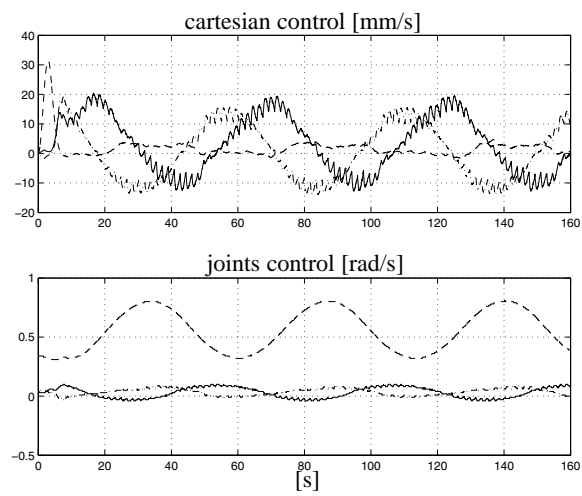

Figure 12: Control signals : $v_{1}(-\cdot-), v_{2}(-), v_{3}(--) ; u_{1}(-\cdot-)$ $, u_{2}(-), u_{3}(--)$.

Remark 3 It is worth mentioning that the area is computed by using a pixel counting method (i.e., zero-order moments). In general this measurements is contaminated by noise. In gray scale mode this is critical because boundary pixels can 


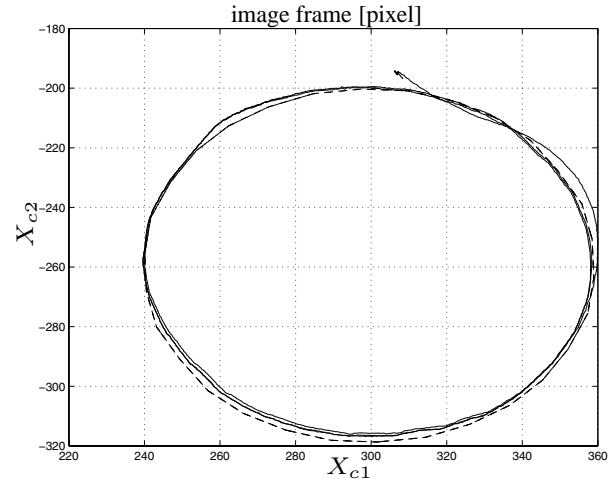

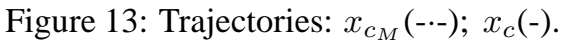

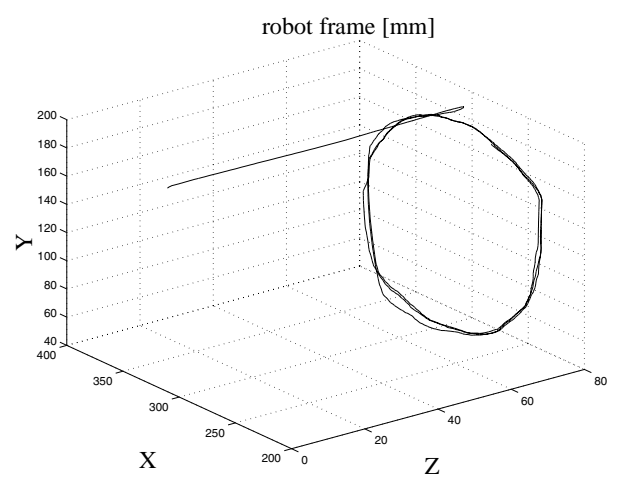

Figure 14: End-effector trajectory.

vary in an unknown pattern even in a well-conditioned environment. In order to overcome this drawback a filtering stage is used to reduce the noise in the area estimate.

\section{CONCLUSION}

In this paper, we have developed an adaptive visual servoing scheme, using an uncalibrated camera, that provides stable $3 \mathrm{D}$ cartesian tracking for robot manipulators without requiring depth measurement. The controller was designed to include the general case of robotic systems in which all the image features were extracted from a spherical object. For the 1D depth subsystem, the MRAC control method was applied, whereas for the multivariable $2 \mathrm{D}$ tracking subsystem, the recently proposed SDU factorization method was adopted. Since a linearly parameterized model was obtained, no explicit inverse image Jacobian estimation was required in the control structure. The stability analysis for the proposed strategy was also presented with global properties. Simulation and experimental results with a real robot manipulator were included to illustrate the performance of the proposed strategy.
Future research topics following the ideas developed here are: navigation of autonomous vehicles using visual servoing, extension to full dynamics robots and application to hybrid vision-force robot control.

\section{REFERENCES}

Conticelli, A. and Allotta, B. (2001a). Discrete-time robot visual servoing in 3-d positioning tasks with depth adaptation, ASME Journal of Dynamic Systems, Measurement and Control 06(3): 356-363.

Conticelli, A. and Allotta, B. (2001b). Nonlinear controlability and stability analysis of adaptive image-based systems, IEEE Transactions on Robotics and Automation 17(2): 208-214.

Corke, P. and Hutchinson, S. (2000). A new hybrid imagebased visual servo control scheme, IEEE Conference on Decision and Control, pp. 2521-2527.

Costa, R. R., Hsu, L., Imai, A. and Kokotovic, P. (2003). Lyapunov-based adaptive control of MIMO systems, Automatica 39(7): 1251-1257.

Espiau, B., Chaumette, F. and Rives, P. (1992). A new approach to visual servoing in robotics, IEEE Transactions on Robotics and Automation 8(3): 313-326.

Fang, Y., Behal, A., Dixon, W. E. and Dawson, D. M. (2002). Adaptive $2.5 \mathrm{~d}$ visual servoing of kinematically redundant robot manipulators, IEEE Conference on Decision and Control, Las Vegas, Nevada, pp. 2860-2865.

Flandin, G., Chaumette, F. and Marchand, E. (2000). Eyein-hand/eye-to-hand cooperation for visual servoing, IEEE International Conference on Robotics \& Automation, San Francisco, CA, pp. 2741-2746.

Haralick, R. M. and Shapiro, L. G. (1993). Computer and Robot Vision, Vol. II, Addison-Wesley.

Hsu, L. and Costa, R. R. (1999). MIMO direct adaptive control with reduced prior knowledge of the high frequency gain, IEEE Conference on Decision and Control, Phoenix, Arizona, pp. 3303-3308.

Hsu, L., Zachi, A. R. L. and Lizarralde, F. (2001). Adaptive visual tracking for motions on smooth surfaces, IEEE Conference on Decision and Control, Orlando, Florida, pp. 1866-1871.

Hutchinson, S., Hager, G. and Corke, P. (1996). A tutorial on visual servo control, IEEE Transactions on Robotics and Automation 12(5): 651-670.

Ioannou, P. and Sun, K. (1996). Robust Adaptive Control, Prentice Hall. 
Kelly, R., Carelli, R., Nasisi, O., Kuchen, B. and Reyes, F. (2000). Stable visual servoing of camera-in-hand robotic systems, ASME Journal of Dynamic Systems, Measurement and Control 15(1): 39-48.

Malis, E., Chaumette, F. and Boudet, S. (1999). 2 1/2D visual servoing, IEEE Transactions on Robotics and Automation 15(2): 238-250.

Ortega, R., Hsu, L. and Astolfi, A. (2003). Immersion and invariance adaptive control of linear multivariable systems, Systems and Control Letters 49: 37-47.

Zachi, A. R. L., Hsu, L. and Lizarralde, F. (2004). Performing stable $2 \mathrm{D}$ adaptive visual positioning/tracking control without explicit depth measurement, IEEE International Conference on Robotics \& Automation, New Orleans, pp. 2297-2302. 